\title{
Using a mechanical spring for monitoring conveyor systems
}

\author{
H. Zur ${ }^{1}$, Ch. Rückerl ${ }^{2}$, K. Eichhorn ${ }^{2}$ \\ ${ }^{1}$ Mechanische Werkstätten Wurzen GmbH, Leipziger Straße 75, \\ 04828 Bennewitz OT Deuben, Germany \\ technik@mw-wurzen.de \\ ${ }^{2}$ FTZ Leipzig, Wächterstraße 13, 04107 Leipzig, Germany
}

\begin{abstract}
:
To guarantee the directional stability of belt conveyor systems the required belt tension has to be ensured. This can be done through mechanical springs. Therefore, a possible sensor concept is shown in this paper. It describes a long-term stable measurement of the belt tension by using the electrical properties of a mechanical spring. The inductance of a spring varies inversely proportional to its length. With measuring the inductance and computing the spring extension, it can be used as a sensor. Initial experiments show that existing belt conveyor systems can be upgraded with this kind of sensor system.
\end{abstract}

Key words: mechanical spring, monitoring, inductance, conveyor systems, belt strain

\section{Motivation}

The monitoring of technical facilities is necessary to identify damages or eliminate malfunctions. It helps to improve the lifespan and ensures the safety of technical facilities. Especially belt conveyor systems are exposed to the weight of the transported goods and external influences. This results in a need for periodical checks.

The belt of a conveyor system has to be prestressed. A non-typical solution is the application of mechanical springs. The pretension is achieved by the spring force. It can be measured by using the springs electrical properties, especially its inductance.

The aim of the sensor system is determining the belt strain by analysing the spring impedance resulting in a monitoring system for conveyor systems that informs about invalid belt tensions. This should help to prevent belt damages.

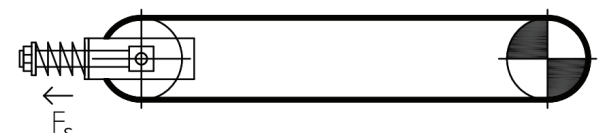

Fig. 1 a.) Pretension with a mechanical spring at the defection roller.

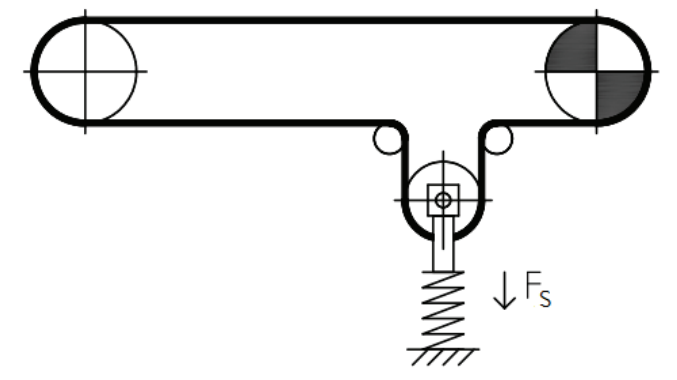

Fig. 1 b.) Pretension with a mechanical spring at the return side

\section{Sensor Principle and Solution}

The inductance of a spring varies in inverse proportion to its length. This means a lower distance between the spring windings results in a higher inductance. By measuring the impedance, the spring can be used as a sensor. To simulate the impedance of a cylindrical spring, a software tool was implemented with particular regard to the computation of the inductance of spiral springs for low frequency problems. It determines the external inductance by computing the vector potential. 


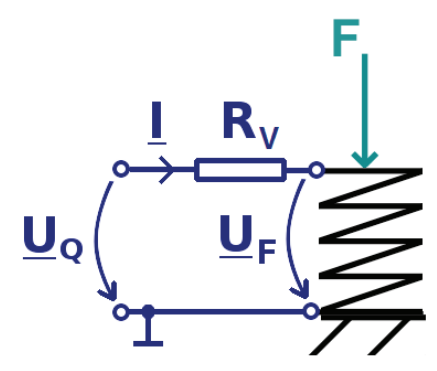

Fig. 2. Sensor principle

$F$ : spring force

I: complex electrical current

Rv: pre-resistor

Uf: complex spring voltage

$\underline{U}_{q}$; complex source voltage

\section{Computation}

To determine the self-inductance of a mechanical spring, one may specify an electrical current, compute the vector potential (eq. 1) along the inner contour (Fig. 3) of the spring path [1] and integrate it along this contour (eq. 2).

$$
\begin{aligned}
& \vec{A}(\vec{r})=\frac{\mu_{0} I}{4 \pi} \int_{c_{1}} \frac{\vec{d} r^{\prime}}{\left|\vec{r}-\vec{r}^{\prime}\right|} \\
& \Phi=\int_{c_{2}} \vec{A}(\vec{r}) \vec{d} r
\end{aligned}
$$

This leads to the value of magnetic flux. In order to obtain the value of external inductance, the magnetic flux has to be divided by the specified current (eq. 3).

$$
L_{e}=\frac{\Phi}{I}
$$

The inner inductance is approximated by

$$
L_{i}=\frac{\mu_{0} \mu_{r} l}{8 \pi}
$$

while $l$ is the length of the wire and $\mu_{r}$ is the relative permittivity of the spring material. The self-inductance $L$ is calculated by summarising the inner inductance $L_{i}$ and the external inductance $L_{e}$.

$L=L_{i}+L_{e}$

The following figure (Fig. 3) illustrates how the external inductance of a single winding is calculated.

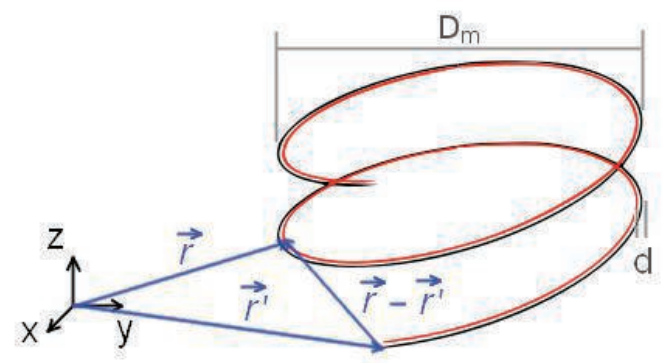

Fig. 3. Computation of the spring inductance $d$ : conductor diameter

Dm: winding diameter

For reasons to do with the calculation, the software uses models that consist of many separate segments. Because of this, the integrals (eq. 1 and eq. 2) are turned into sums. The number of segments depends on the number of windings as every winding is divided into a constant amount of parts.

This kind of calculation has, of course, its limitations. First, it requires that the spring be enclosed in a homogeneous, non-conducting medium. Second, the inductance results are incorrect when it comes to high frequency problems, because skin effect and proximity effect are not considered here. Furthermore, a non-ferromagnetic spring material is expected.

\section{Results}

The following example shows the influence of the spring deflection to its inductance and its impedance. Therefore, a spring with the following parameters was used for measurement and simulation.

Tab. 1: Spring parameters

\begin{tabular}{|c|c|}
\hline Parameter & Value / condition \\
\hline Spring length & $150 \mathrm{~mm}$ \\
\hline Winding diameter & $42,5 \mathrm{~mm}$ \\
\hline Wire diameter & $2 \mathrm{~mm}$ \\
\hline Number of windings & 11,75 \\
\hline Ends of the spring & $\begin{array}{c}\text { Hooked together, } \\
\text { not planed }\end{array}$ \\
\hline Material & Spring steel 1.4310 \\
\hline
\end{tabular}

Figure 4 shows the inductance results of the software simulation and the measurement. Therefore, a FLUKE PM6306 impedance meter was used. This device displays the calculated inductance of an equivalent circuit diagram that consists of a resistor and an ideal inductance. It means the spring capacitance is not displayed 
separately but affects both results. That leads to the three frequency dependend inductance graphs. For a frequency of $50 \mathrm{kHz}$ there is a very good congruency between simulation and the measured data.

Furthermore, the impedance was simulated for these frequencies. The graphs are presented in figure 3. For lower excitation frequencies, there is a good congruency. As the software tool ignores the capacitance of the spring, the divergences between the simulation results and the measured data are bigger for increasing frequencies

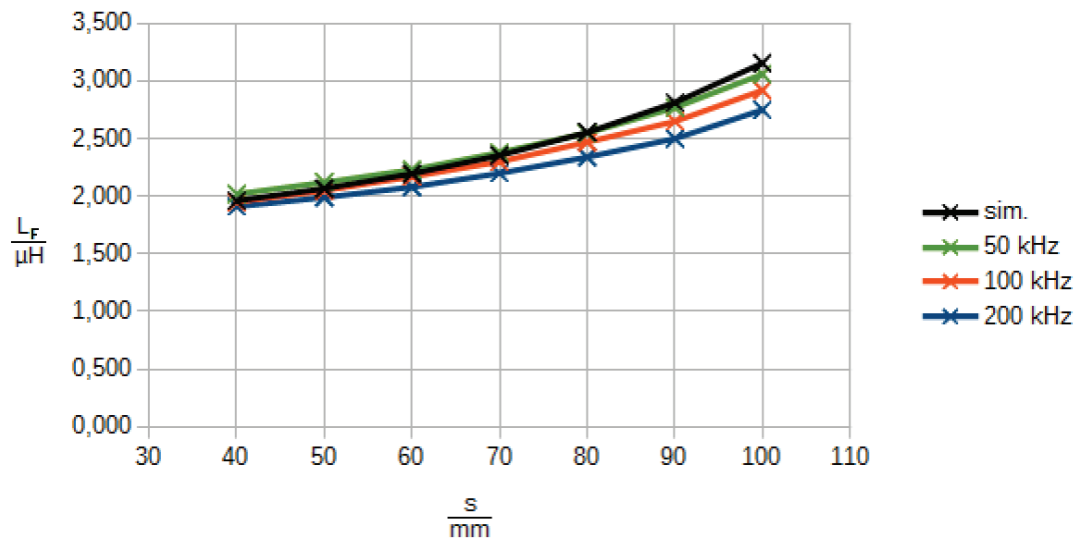

Fig. 4. Inductance results of simulation and measurement black: results of the simulation green: measurement results with an excitation frequency of $50 \mathrm{kHz}$ red: measurement results with an excitation frequency of $100 \mathrm{kHz}$ blue: measurement results with an excitation frequency of $200 \mathrm{kHz}$

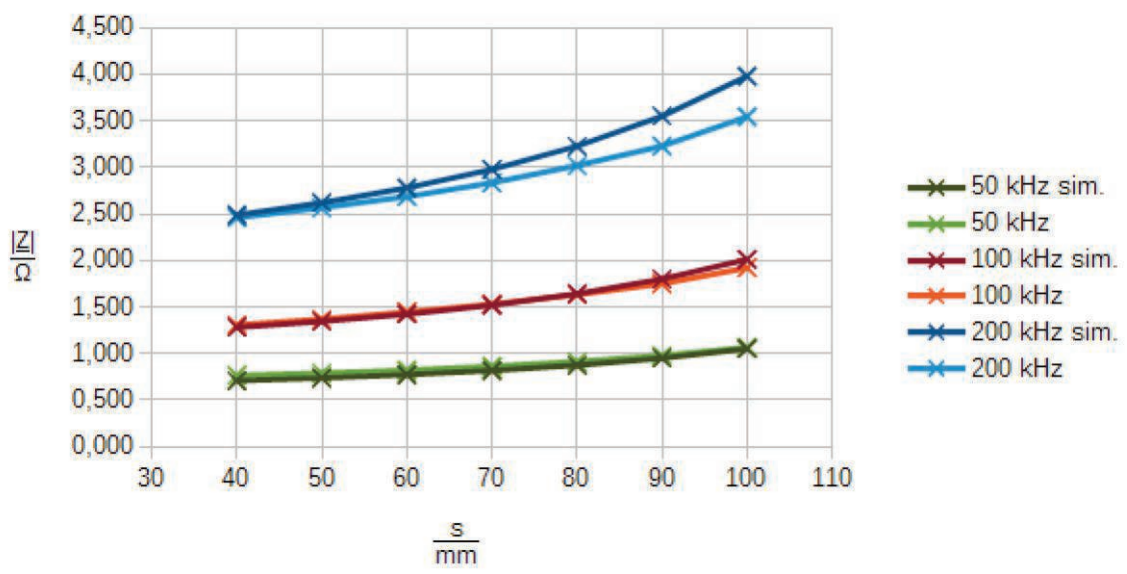

Fig. 5. Impedance results of simulation and measurement green: measurement results with an excitation frequency of $50 \mathrm{kHz}$ dark green: simulation results with an excitation frequency of $50 \mathrm{kHz}$ red: measurement results with an excitation frequency of $100 \mathrm{kHz}$ dark red: simulation results with an excitation frequency of $100 \mathrm{kHz}$ blue: measurement results with an excitation frequency of $200 \mathrm{kHz}$ dark blue: simulation results with an excitation frequency of $200 \mathrm{kHz}$

\section{References}

[1] P. Römisch, Materialflusstechnik, Vieweg + Teubner (2011)

[2] K. Küpfmüller, G. Kohn, Theoretische Elektrotechnik ed 14, Springer (1993)

[3] K. Fr. Eichhorn, Induktivitäten windschiefer Leitersysteme, Electrical Engineering Vol. 61, No. 6 (1979) 